\title{
Binding entanglement channels
}

\author{
Paweł Horodecki*, Michał Horodecki ${ }^{2}$,* Ryszard Horodecki2 ${ }^{2 * *}$ \\ ${ }^{1}$ Faculty of Applied Physics and Mathematics, Technical University of Gdańsk, 80-952 Gdańsk, Poland \\ ${ }^{2}$ Institute of Theoretical Physics and Astrophysics, University of Gdańsk, 80-952 Gdańsk, Poland
}

We define the binding entanglement channel as the quantum channel through which quantum information cannot be reliably transmitted, but which can be used to share bound entanglement. We provide a characterization of such class of channels. We also show that any bound entangled state can be used to construction of the map corresponding the binding entanglement channel.

\section{INTRODUCTION}

One of the recent results leading to better understanding of quantum entanglement [1]:2 was realizing that there are two qualitatively different types of entanglement of mixed states of two-component systems [3, 4. Namely, there is free entanglement (FE) which can be converted into pure singlet form by means of local quantum operations and classical communication (LQCC). Such a process is called distillation [5] and it allows to use the noisy entanglement for the purposes of quantum communication. However, there is also bound entanglement (BE), which cannot be distilled [3, 4. At present the structure and properties of $\mathrm{BE}$ state are being extensively investigated [6,7,9 12]. In particular, a striking connection between the bound etanglement and nonlocality without entanglement [8] has been discovered [9]. Also, the bound entanglement implies a new approach in entanglement measures: one must, in general leave the paradigm that a measure of entanglement should vanish only on separable states. Indeed, at present we know that physically the most relevant measure of entanglement 1315 which is distillable entanglement does not satisfy this condition (it vanishes on the bound entangled states). The above, more general approach allowed to obtain a new bound on distillable entanglement Ref. [12. Due to the connection between entanglement and positive maps [16] the investigation of bound entanglement was also fruitful for pure mathematics. Namely, by use of results on bound entanglement of Ref. [9] the first systematic way of constructing the so called non-decomposable positive maps was found in Ref. [10].

In this paper we would like to investigate the processes of interaction with environment, which lead to bound entanglement. In general, the mixed states emerge from interaction with environment, which is very hard to be avoided in realistic situation. Such interaction may completely destroy the initial pure entanglement, or sometimes there may remain some residual entanglement, free or bound. We will be interested in the processes for which the residual entanglemet is the bound one. To be more precise, imagine that Alice can send particles to Bob via a quantum channel $\Lambda$ (representing the interaction with environment). Alice and Bob are allowed to support the quantum channel by using LQCC operations and can enhance the transmission by sending entangled particles down the channel. The latter means that effectively they have a channel $\Lambda^{\otimes N}$ for arbitrary $N$. Now we are interested in such channels that Alice and Bob (i) cannot send reliably quantum information (equivalently, cannot produce asymptotically singlet state); (ii) can produce a BE state. Such channels we will call binding entanglement channels (BE channels).

We prove a theorem characterizing such channels, which says that a channel is BE if and only if sending half of maximally entangled pair through the channel, one obtains $\mathrm{BE}$ state. It follows that a channel is $\mathrm{BE}$ if there exists a pure entangled state such, that if sent through the channel it becomes bound entangled. Thus knowing the examples of BE states, we can construct the BE channels. We provide a way of constructing BE channel from any given BE state. Our investigations are based on the general connections between channels and bipartite states investigated in $17,13,18,19,11$.

\section{BINDING ENTANGLEMENT CHANNELS: CHARACTERIZATION}

To begin with, let us introduce some notation. By a channel we mean any completely positive (CP) tracepreserving map. A completely positive map $\Lambda: M_{m} \rightarrow$ $M_{n}$ will be denoted by $\Lambda_{m}^{n}$ (here $M_{n}$ denotes the set of $n \times n$ square matrices. The identity map acting on $M_{n}$ will be denoted by $I_{n}$. Maximally entangled state on the system $M_{n} \otimes M_{n}$ of the form

$$
P_{+}^{n}=\frac{1}{\sqrt{n}} \sum_{i=1}^{n}|i\rangle|i\rangle
$$

will be called singlet state. A state acting on the Hilbert space $C^{m} \otimes C^{n}$ will be denoted by $\varrho_{m, n}$ (or $\sigma_{m, n}$ etc.). Sometimes, if it does not lead to misunderstanding we will not write the indices explicitly. Finally, $\varrho_{i k j l}$ denotes matrix element of the state $\varrho$ in product basis

$$
\varrho_{i k j l} \equiv\left\langle e_{i} \otimes f_{k}|\varrho| e_{j} \otimes f_{l}\right\rangle
$$

Definition. We say that a channel $\Lambda$ is binding entanglement channel iff (i) $Q_{2}(\Lambda)=0$ and (ii) it is possible to 
obtain bipartite bound entangled state by means of (possibly multiply) use of the channel and LQCC operations.

Here $Q_{2}$ is the quantum capacity of a channel supported by LQCC action (the subscript 2 indicates twoway classical communication) 13]. Now we will prove a theorem characterizing such channels in terms of $\mathrm{BE}$ bipartite states.

Theorem. A channel $\Lambda: M_{m} \rightarrow M_{n}$ is binding entanglement iff the state $(I \otimes \Lambda) P_{+}^{m}$ (acting on $C^{m} \otimes C^{n}$ ) is a $\mathrm{BE}$ state.

Proof. Let us first prove the sufficiency of the condition. If $\left(I \otimes \Lambda_{m}^{n}\right) P_{+}^{m}$ is BE state then (ii) is obviously satisfied, so that one needs to prove that the condition implies also (i). Suppose, conversely, that $Q_{2}\left(\Lambda_{m}^{n}\right)>0$. Then, one can produce asymptotically pure singlets by use of the channel and LQCC. The first stage of the most general protocol of producing singlet pairs is sending half of some state $\sigma_{k \times N, m \times N}$ via the channel $\Lambda^{\otimes N}$ (denote it by $\Lambda_{m \times N}^{n \times N}$ ). The second stage amounts to distillation of the emerging state $\varrho_{k \times N, m \times N}=\left(I_{k \times N} \otimes \Lambda_{m \times N}^{n \times N}\right) \sigma$. Hence, to obtain finally the singlets, the state $\varrho$ must be FE. We will now show that this implies that $\left(I_{m} \otimes \Lambda_{m}^{n}\right) P_{+}^{m}$ must be also $\mathrm{FE}$. To see it, note that the state $\sigma$ (as any state) can be written as $\sigma=\left(\Gamma_{m \times N}^{k \times N} \otimes I_{m \times N}\right) P_{+}^{m \times N}$ (where $\Gamma$ is $\mathrm{CP}$, but not necessarily trace-preserving map). So we have

$$
\begin{aligned}
& \varrho=\left(I_{k \times N} \otimes \Lambda_{m \times N}^{n \times N}\right)\left(\Gamma_{m \times N}^{k \times N} \otimes I_{m \times N}\right) P_{+}^{m \times N}= \\
& \left(\Gamma_{m \times N}^{k \times N} \otimes \Lambda_{m \times N}^{n \times N}\right) P_{+}^{m \times N}= \\
& \left(\Gamma_{m \times N}^{k \times N} \otimes I_{n \times N}\right)\left(I_{m \times N} \otimes \Lambda_{m \times N}^{n \times N}\right) P_{+}^{m \times N}
\end{aligned}
$$

Now, since $\varrho$ is FE, then also $\left(I_{m \times N} \otimes \Lambda_{m \times N}^{n \times N}\right) P_{+}^{m \times N}$ must be FE (indeed the action $\Gamma \otimes I$ is LQCC one, hence cannot produce $\mathrm{FE}$ state from a $\mathrm{BE}$ one). Now, since $\left(I_{m \times N} \otimes \Lambda_{m \times N}^{n \times N}\right) P_{+}^{m \times N}=\left(\left(I_{m} \otimes \Lambda_{m}^{n}\right) P_{+}^{m}\right)^{\otimes N}$ we obtain that also $\left(I_{m} \otimes \Lambda_{m}^{n}\right) P_{+}^{m}$ must be FE, which is a contradiction. Hence, if $\left(I_{m} \otimes \Lambda_{m}^{n}\right) P_{+}^{m}$ is BE then the condition (i) is satisfied.

Now, we will show that the condition that $\left(I_{m} \otimes\right.$ $\left.\Lambda_{m}^{n}\right) P_{+}^{m}$ is BE is also a necessary one for $\Lambda$ to be BE. Suppose, conversely, that $\left(I_{m} \otimes \Lambda_{m}^{n}\right) P_{+}^{m}$ is not BE. Then it can be separable or FE. If its is FE, then one can distill it and obtain nonzero $Q_{2}$ so that the condition (i) is violated. If, instead $\left(\Lambda_{m}^{n} \otimes I_{m}\right) P_{+}^{m}$ is separable, then we will show that the condition (ii) is violated. Indeed, if for some state $\sigma_{k, m}$ the state $\varrho_{m, n}=\left(I_{m} \otimes \Lambda_{m}^{n}\right) \sigma_{k, m}$ is $\mathrm{BE}$, then writing $\sigma$ as $\sigma_{k, m}=\left(\Gamma_{k}^{m} \otimes I_{m}\right) P_{+}^{m}$ we obtain, similarly as in the proof of sufficiency, that $\varrho=$ $\left(\Gamma_{k}^{m} \otimes I_{n}\right)\left(I_{m} \otimes \Lambda_{m}^{n}\right) P_{+}^{m}$. Then, since $\Gamma \otimes I$ is LQCC, we obtain that $\left(I_{m} \otimes \Lambda_{m}^{n}\right) P_{+}^{m}$ cannot be separable (LQCC action cannot make BE state from separable one). This ends the proof.

From the above characterization of BE channels it follows that given a channel with $Q_{2}=0$, if bound entanglement can be created at all, then it can be created with- out exchange of classical information between Alice and Bob but merely by sending half of singlet pair through the channel. Hence also multiply use of channel is not needed.

\section{BININDG ENTANGLEMENT CHANNELS FROM BOUND ENTANGLED STATES}

In this section we will provide a procedure of constructing $\mathrm{BE}$ channels from BE states. As one knows there is an isomorphism between the set of states $\varrho_{m, n}$ with maximally mixed reduction $\varrho_{A}$ and the channels $\Lambda_{m}^{n}$. It is given just by the formula:

$$
\varrho_{m, n}=\left(I_{m} \otimes \Lambda_{m}^{n}\right) P_{+}^{m}
$$

(the maximally mixed reduction is connected with the fact that channels preserve trace). In other words, if one has a channel, one can send half of singlets through it to obtain the state with maximally mixed reduction, and, conversely, any state of maximally mixed reduction emerges from sending half of singlet down some channel. Explicitly, the connection between matrix elements of state and associated channel is the following

$$
\left\langlef _ { k } \left|\Lambda \left(\left|e_{i}\right\rangle\left\langle e_{j}\right)\left|f_{l}\right\rangle \equiv \lambda_{k l i j}=\varrho_{i k j l} \equiv\left\langle e_{i} \otimes f_{k}|\varrho| e_{j} \otimes f_{l}\right\rangle .\right.\right.\right.
$$

So we can provide examples of BE channels basing on the known BE states with maximally mixed reduction. However, one also knows the examples of BE states with none of reductions maximally mixed [9]. How to associate channels with them? As mentioned above, the maximally mixed reduction is connected with the fact that the channel acts only on one half of the singlet, so that, being trace-preserving, it cannot disturb the other one. Since the singlet is maximally entangled, it has maximally mixed reduction that is inherited by the final state. Now, if a state with non-maximally mixed reductions is concerned, one can imagine it emerges from sending nonmaximally entangled pure state via a channel. The state must have the same reduction as the mixed state of interest (as, again, the channel will not affect that reduction). To recover such a channel from the given state $\varrho$, we will first transform it into a state $\sigma$ of maximally mixed reduction by means of LQCC action. Then the channel will be the one associated with $\sigma$ via the state-channel isomorphism (3). Let a BE state $\varrho_{m, n}$ acts on $\mathcal{H}_{A} \otimes \mathcal{H}_{B}$ and let $\mathcal{H}_{A}^{\prime}$ be the support of its reduction $\varrho_{A}$ with $\operatorname{dim} \mathcal{H}_{A}^{\prime}=k$. Then define

$$
\sigma_{r, n}=\left(r \varrho_{A}\right)^{-1 / 2} \otimes I \varrho\left(r \varrho_{A}\right)^{-1 / 2} \otimes I,
$$

where $\varrho_{A}$ was inverted on its support $\mathcal{H}_{A}^{\prime}$ Here we used the fact that the support of any state is equal to product of the supports of its reductions (see Appendix), so that, 
in fact, both $\varrho$ and $\sigma$ acts on $\mathcal{H}_{A}^{\prime} \otimes \mathcal{H}_{B}$. It is easy to check that $\sigma_{A}=\frac{I}{r}$. Indeed, choosing the basis $\left\{e_{i}\right\} \subset \mathcal{H}_{A}^{i}$ to be eigenbasis of $\varrho_{A}$ (i.e. $\varrho_{A}=\sum_{i} p_{i}\left|e_{i}\right\rangle\left\langle e_{i}\right|$ ) we obtain

$$
\sigma_{i k j l}=\frac{1}{r \sqrt{p_{i} p_{j}}} \varrho_{i k j l}
$$

hence

$$
\left(\sigma_{A}\right)_{i j}=\sum_{k} \sigma_{i k j k}=\frac{1}{r \sqrt{p_{i} p_{j}}} p_{i} \delta_{i j}=\frac{1}{r} \delta_{i j}
$$

Now, as the state $\sigma$ was created from $\varrho$ by LQCC action, then it is $\mathrm{BE}$ (the action is called filtering [20]). Then the seeked BE channel $\Lambda_{A}$ corresponding to the given state $\varrho$ is the one associated with the state $\sigma$ via the formula (4) (the subscript $A$ indicates that we recover the channel by use of the reduction $\varrho_{A}$ ).

Then to obtain explicit form of $\Lambda_{A}$ one needs to calculate the map $\Theta$ given by the formula

$$
\left(I_{r} \otimes \Theta_{r}^{n}\right) P_{+}^{r}=\varrho .
$$

Then $\Lambda_{A}$ is given by

$$
\Lambda_{A}=\Theta \circ \Gamma_{A}^{T}
$$

where $\Gamma_{A}(\cdot)=\frac{1}{r} \varrho_{A}^{-1 / 2}(\cdot) \varrho_{A}^{-1 / 2}$ and $T$ is tranpose in the space of maps i.e. $\left(\Theta_{B}^{T}\right)_{k l i j}=\left(\Theta_{B}\right)_{i j k l}$. If the given map $\Lambda$ is CP (as in our case) and its Stinespring form is $\Lambda(\cdot)=\sum_{i} V_{i}(\cdot) V_{i}^{\dagger}$ then the transposed map is given simply by $\Lambda(\cdot)=\sum_{i} V_{i}^{T}(\cdot)\left(V_{i}^{T}\right)^{\dagger}$. Thus we obtain that

$$
\Lambda_{A}(\cdot)=\frac{1}{r} \Theta\left(\left(\varrho_{A}^{T}\right)^{-1 / 2}(\cdot)\left(\varrho_{A}^{T}\right)^{-1 / 2}\right) .
$$

Note that if only $\varrho_{A}$ is not maximally mixed then both $\Gamma$ and $\Theta$ are not trace-preserving. Nevertheless $\Lambda$ is tracepreserving so that it constitutes a channel.

Of course one can use the other reduction of the state $\varrho$ to obtain a channel (call it $\Lambda_{B}$ ). Then one can get the following formula

$$
\Lambda_{B}(\cdot)=\frac{1}{r} \Theta^{T}\left(\left(\varrho_{B}^{T}\right)^{-1 / 2}(\cdot)\left(\varrho_{B}^{T}\right)^{-1 / 2}\right)
$$

Now, the state $\varrho$ emerges if (i) Alice send to Bob some pure state $\psi$ of both reductions equal to $\varrho_{A}$ via the channel $\Lambda_{A}$, or (ii) Bob sends to Alice the pure state of reductions $\varrho_{B}$ through the channel $\Lambda_{B}$.

\section{EXAMPLES}

A simple way of recovering the maps from a given state via formula (8) is to use the eigenbasis of the state. Namely, if

$$
\varrho_{m, n}=\sum_{i} p_{i}\left|\psi_{i}\right\rangle\left\langle\psi_{i}\right|
$$

with

$$
\psi_{i}=\sum_{j=1}^{m} \sum_{k=1}^{n} c_{j, k}^{i}\left|e_{i}\right\rangle\left|f_{i}\right\rangle,
$$

then the associated map $\Theta_{m}^{n}$ is given by

$$
\Theta(\cdot)=\sum_{i} p_{i} V_{i}(\cdot) V_{i}^{\dagger}
$$

with $\left\langle e_{j}\left|V_{i}\right| f_{k}\right\rangle=m c_{j, k}^{i}$. If it is hard to find the eigenbasis, one can use any decomposition of the BE state into pure ones.

Example 1. In the paper Ref. [3] we modified the Størmer 21] matrix to obtain the following family of twoqutrit BE states

$$
\sigma_{\alpha}=\frac{2}{7} P_{+}^{3}+\frac{\alpha}{7} \sigma_{+}+\frac{5-\alpha}{7} \sigma_{-},
$$

where $3<\alpha \leq 4$ and

$$
\begin{gathered}
\sigma_{+}=\frac{1}{3}(|0\rangle|1\rangle\langle 0|\langle 1|+| 1\rangle| 2\rangle\langle 1|\langle 2|+| 2\rangle| 0\rangle\langle 2|\langle 0|), \\
\sigma_{-}=\frac{1}{3}(|1\rangle|0\rangle\langle 1|\langle 0|+| 2\rangle| 1\rangle\langle 2|\langle 1|+| 0\rangle| 2\rangle\langle 0|\langle 2|) .
\end{gathered}
$$

The above state has both reductions maximally mixed, so that we could consider two channels $\left(\varrho=\left(I \otimes \Lambda_{1}\right) P_{+}\right.$ and $\left.\varrho=\left(\Lambda_{2} \otimes I\right) P_{+}\right)$. However, due to symmetry of the state, the two cases give raise to the same family of channels, given by

$$
\begin{aligned}
& \Lambda(\cdot)=\frac{2}{7}(\cdot)+\frac{\alpha}{7} \sum_{k=1}^{3} P_{k \oplus 1 k}(\cdot) P_{k k \oplus 1}+ \\
& \frac{5-\alpha}{7} \sum_{k=1}^{3} P_{k \ominus 1 k}(\cdot) P_{k k \ominus 1}
\end{aligned}
$$

where $P_{i j}=|i\rangle\langle j| ; \oplus$ and $\ominus$ denote + and - modulo 3 respectively.

Example 2. This example will be based on the twoqutrit BE state [4] of the following form

$$
\varrho=\frac{1}{8 a+1}\left[\begin{array}{ccccccccc}
a & 0 & 0 & 0 & a & 0 & 0 & 0 & a \\
0 & a & 0 & 0 & 0 & 0 & 0 & 0 & 0 \\
0 & 0 & a & 0 & 0 & 0 & 0 & 0 & 0 \\
0 & 0 & 0 & a & 0 & 0 & 0 & 0 & 0 \\
a & 0 & 0 & 0 & a & 0 & 0 & 0 & a \\
0 & 0 & 0 & 0 & 0 & a & 0 & 0 & 0 \\
0 & 0 & 0 & 0 & 0 & 0 & \frac{1+a}{2} & 0 & \frac{\sqrt{1-a^{2}}}{2} \\
0 & 0 & 0 & 0 & 0 & 0 & 0 & a & 0 \\
a & 0 & 0 & 0 & a & 0 & \frac{\sqrt{1-a^{2}}}{2} & 0 & \frac{1+a}{2}
\end{array}\right],
$$

where $0<a<1$. The reduction $\varrho_{A}$ of the state is given by 


$$
\varrho_{A}=\frac{1}{8 a+1}\left[\begin{array}{ccc}
3 a & 0 & 0 \\
0 & 3 a & 0 \\
0 & 0 & 1+2 a
\end{array}\right]
$$

hence it is not maximally mixed. Now, to recover the channel we can apply the formula (10). The map $\Gamma$ is given by

$$
\begin{gathered}
\Gamma(\cdot)=\frac{a}{8 a+1}\left(3(\cdot)+P_{12}(\cdot) P_{21}+P_{13}(\cdot) P_{31}+P_{21}(\cdot) P_{12}+\right. \\
\left.P_{23}(\cdot) P_{32}+P_{32}(\cdot) P_{23}\right)+\frac{1}{8 a+1} W(\cdot) W^{\dagger}
\end{gathered}
$$

where $W=\sqrt{\frac{1+a}{2}} P_{31}+\sqrt{\frac{1-a}{2}} P_{33}$. Since $\varrho_{A}$ is diagonal we obtain $\Gamma_{A}^{T}=\Gamma_{A}$ Then the final form of the channel $\Lambda_{A}$ is given by

$$
\begin{aligned}
& \Lambda_{A}(\cdot)=\frac{a}{3}\left(3 V(\cdot) V+\frac{1}{3 a}\left(P_{12}(\cdot) P_{21}+P_{32}(\cdot) P_{23}\right.\right. \\
& \left.\left.+P_{21}(\cdot) P_{12}\right)+\frac{1}{2 a+1}\left(P_{13}(\cdot) P_{31}+P_{23}(\cdot) P_{32}\right)\right)+\tilde{W}(\cdot) \tilde{W}^{\dagger}
\end{aligned}
$$

where $V=\operatorname{diag}[1 / \sqrt{3 a}, 1 / \sqrt{3 a}, 1 / \sqrt{2 a+1}]$ and $\tilde{W}=$ $\sqrt{\frac{1+a}{6 a}} P_{31}+\sqrt{\frac{1-a}{2(2 a+1)}} P_{33}$.

\section{DISCUSSION}

Let us now discuss some possible directions of further investigation of the binding entnaglement channels. The main goal will be to find how the BE channels could be useful for quantum communication. The hint is given by the effect of activation of bound entanglement [6], where a large amount of $\mathrm{BE}$ systems considerably raised the possibilities of a single FE system. In Ref. [6] we rose a question, whether the channels associated with the BE states (which, due to theorem, are BE channels) could exhibit nonadditivity in the following sense. If we have a channel of some nonzero capacity $Q$, and a $\mathrm{BE}$ channel, then by using the channels jointly, one expects to obtain total capacity greater than $Q$.

Another question arises, if we consider the BE channel as public one (cf. [6]). This changes the paradigm of entanglement manipulations, where so far, only classical communication was public. The question is: what is capacity of some quantum channel of nonzero standard capacity (either with or without classical comunication) if supplemented with public BE channel? It was natural to expect that the capacity of the supported channel could be strictly greater, especially, because, as reported in Ref. [9], the BE states can have surprisingly large entanglement of formation $\left(E_{f}\right)$. The two-qutrit states provided in Ref. [9] have $E_{f} \simeq 0.2$ of entanglement of formation while the maximally entangled state of two-qutrits has $E_{f} \simeq 1.5$. Now we would like to ask the following question: may be, the channel supported by public BE channel have maximal capacity, determined only by the Hilbert space of the sent systems? This could be called the effect of cristallization of bound entanglement. The conjecture is not unreasonable: we have, in fact, infinite amount of bound entanglement at our disposal.

\section{Appendix}

Here we prove the following lemma:

Lemma. The support of any state is included in the product of the supports of the reductions of the state

$$
\operatorname{supp} \varrho \subseteq \operatorname{supp} \varrho_{A} \otimes \operatorname{supp} \varrho_{B}
$$

Proof. Let us first prove the lemma for pure state $|\psi\rangle\langle\psi|$. Writing the state in the Schmidt decomposition we see that it is a superposition of the products of the states belonging to supports of the reductions, so that the thesis of the lemma holds. Now, for the mixed state $\stackrel{\varrho}{(21)}=\sum_{i}\left|\psi_{i}\right\rangle\left\langle\psi_{i}\right|$ we have

$$
\operatorname{supp} \varrho=\operatorname{span}\left\{\psi_{i}\right\}_{i}
$$

and

$$
\operatorname{supp} \varrho_{A} \otimes \operatorname{supp} \varrho_{B}=\operatorname{span}\left\{\operatorname{supp} \varrho_{A}^{i} \otimes \operatorname{supp} \varrho_{B}^{i}\right\}_{i},
$$

where $\varrho_{A, B}^{i}$ are the reductions of the states $\psi_{i}$. Hence we obtain the required inclusion.

* $\quad$ Electronic address: michalh@iftia.univ.gda.pl

** Electronic address: pawel@mifgate.pg.gda.pl

*** Electronic address: fizrh@univ.gda.pl

[1] A. Einstein, B. Podolsky and N. Rosen, Phys. Rev. 47, 777 (1935).

[2] E. Schrödinger, Natürwissenschaften 23, 807 (1935).

[3] M. Horodecki, P. Horodecki, and R. Horodecki, Phys. Rev. Lett. 80, 5239 (1998).

[4] P. Horodecki, Phys. Lett. A 232, 233 (1997).

[5] C. H. Bennett, G. Brassard, S. Popescu, B. Schumacher, J. Smolin and W. K. Wootters, Phys. Rev. Lett. 76, 722 (1996).

[6] P. Horodecki, M. Horodecki, and R. Horodecki, Phys. Rev. Lett. 82, 1046 (1999).

[7] N. Linden and S. Popescu, Phys. Rev. A 59, 137 (1999).

[8] C. H. Bennett, D. DiVincenzo, Ch. Fuchs, T. Mor, P. Shor, J. Smolin, E. Rains, and W. K. Wooters, Phys. Rev. A 59, 1070 (1999).

[9] Ch. Bennett, D. DiVincenzo, T. Mor, P. Shor, J. Smolin, and B. Terhal quant-ph/9808030.

[10] B. Terhal, quant-ph/9810091.

[11] M. Horodecki, P. Horodecki and R. Horodecki, quantph/9807091 (Phys. Rev. A, in press).

[12] E. Rains, quant-ph/9809082. 
[13] C. H. Bennett, D. P. DiVincenzo, J. Smolin, and W. K. Wootters, Phys. Rev. A 54, 3824 (1996).

[14] S. Popescu and D. Rohrlich, Phys. Rev. A 56, 3319 (1997).

[15] V. Vedral, M. B. Plenio, M. A. Rippin, and P. L. Knight, Phys. Rev. Lett. 78, 2275 (1997); V. Vedral and M. Plenio, Phys. Rev. A 57, 1619 (1998).

[16] M. Horodecki, P. Horodecki and R. Horodecki Phys. Lett. A 223, 1 (1996).

[17] A. Jamiołkowski, Rep. Math. Phys. 3, 275 (1972).

[18] B. Schumacher and M. A. Nielsen, Phys. Rev. A 54, 2629 (1996); S. Lloyd, ibid. 55, 1613 (1997).

[19] M. Horodecki and P. Horodecki, quant-ph/9708015, Phys. Rev. A, in press.

[20] N. Gisin, Phys. Lett. A 210, 151 (1996); C. H. Bennett, H. Bernstein, S. Popescu and B. Schumacher, Phys. Rev. A 53, 2046 (1996);

[21] E. Størmer, Proc. Amer. Math. Soc. 86, 402 (1982). 